\title{
Limits to mode-localized sensing using micro- and nanomechanical resonator arrays
}

\author{
P. Thiruvenkatanathan, ${ }^{1,2}$ J. Woodhouse, ${ }^{1}$ J. Yan, ${ }^{1,2}$ and A. A. Seshia ${ }^{1,2, a)}$ \\ ${ }^{1}$ Department of Engineering, University of Cambridge, Trumpington Street, Cambridge CB2 IPZ, \\ United Kingdom \\ ${ }^{2}$ Nanoscience Centre, University of Cambridge, 11 J. J. Thomson Avenue, Cambridge CB3 OFF, \\ United Kingdom
}

(Received 15 October 2010; accepted 10 April 2011; published online 18 May 2011)

\begin{abstract}
In recent years, the concept of utilizing the phenomenon of vibration mode-localization as a paradigm of mechanical sensing has made profound impact in the design and development of highly sensitive micro- and nanomechanical sensors. Unprecedented enhancements in sensor response exceeding three orders of magnitude relative to the more conventional resonant frequency shift based technique have been both theoretically and experimentally demonstrated using this new sensing approach. However, the ultimate limits of detection and in consequence, the minimum attainable resolution in such mode-localized sensors still remain uncertain. This paper aims to fill this gap by investigating the limits to sensitivity enhancement imposed on such sensors, by some of the fundamental physical noise processes, the bandwidth of operation and the noise from the electronic interfacial circuits. Our analyses indicate that such mode-localized sensors offer tremendous potential for highly sensitive mass and stiffness detection with ultimate resolutions that may be orders of magnitude better than most conventional micro- and nanomechanical resonant sensors. (C) 2011 American Institute of Physics. [doi:10.1063/1.3590143]
\end{abstract}

\section{INTRODUCTION}

Over the past decade, resonant sensing has emerged as a promising technique for highly sensitive detection of small, linear parametric variations in the structural properties of micro- and nanomechanical sensors. The naturally high frequency sensitivity of such micron/sub-micron devices ${ }^{1,2}$ and the quasidigital nature of the output signal ${ }^{3}$ have made this sensing paradigm particularly attractive for a wide range of applications including bio-molecular ${ }^{4}$ and chemical mass sensing, ${ }^{5}$ acceleration ${ }^{6}$ and strain sensing ${ }^{7}$ among several others. In most of these sensors, the output signal corresponds to a measure of the relative shift in resonant frequency of a vibrating micro- or nanomechanical structure that is subjected to small induced perturbations in structural properties (mass and/or stiffness).

In contrast, the concept of measuring shifts in the eigenstates (i.e., the normalized mode shapes) due to vibration localization in arrays of weakly coupled micro- or nanomechanical resonators has also been proposed as an alternate sensing mechanism in recent years. ${ }^{8-10}$ Besides its high sensitivity to structural perturbations, an eigenmode-shift based sensor (or more simply, a mode-localized sensor), also offers the added advantage of intrinsic common mode rejection that makes it less susceptible to false positive outputs arising from ambient environmental fluctuations. ${ }^{11}$ Parametric sensitivities that are orders of magnitude greater than corresponding resonant frequency shifts have been both theoretically and experimentally demonstrated using this new paradigm of mechanical sensing consequently making such sensors excel-

\footnotetext{
a) Author to whom all correspondence should be addressed. Electronic mail: aas41@cam.ac.uk.
}

lent candidates for a wide variety of sensing applications. The concept has now been successfully implemented for ultrasensitive inertial mass sensing by subjecting weakly coupled, nearly identical micro- or nanomechanical resonators to minute symmetry breaking, differential mass additions and measuring the induced variation in the eigenmodes. The measured shifts in mode shapes have been demonstrated to be not only dependent on the magnitude of induced mass perturbation but also on the strength of internal coupling between the resonators consequently resulting in mass sensitivities that are as high as two to three orders of magnitude greater than corresponding resonant frequency variations. ${ }^{8,9,12}$ These results while establishing a new avenue of mechanical sensing also suggest possible extensions of the technology for the development of a new class of bio-molecular and chemical mass sensors with ultrahigh parametric sensitivity. More recently, the concept has also been utilized for monitoring small changes in the elastic stiffness of vibrating micromechanical resonators thereby extending the applicability of this new paradigm to the mechanical sensing of strain and inertial forces. ${ }^{10,11}$

While the results elaborated in prior work $^{8-12}$ clearly demonstrate significant enhancements in the system response of such mode-localized sensors relative to their resonant frequency shift based counterparts (i.e., an enhancement in the responsivity or the sensitivity of the system to an induced perturbation), their ultimate resolution depend critically on the short-term and long-term amplitude stability of the coupled resonator platforms at their fundamental modes of vibration. Just as in the case of resonant sensors, the output response stability in mode-localized sensors is governed by two disparate classes of noise mechanisms-extrinsic noise processes arising from the external electronic interfacial readout circuitry, and intrinsic noise processes that are 
inherent to the micro- or nanomechanical resonator arrays that serve as platforms for mode-localized sensing. In this paper, we evaluate the impact of some of these intrinsic and extrinsic noise processes on the ultimate resolution of such mode-localized sensors. In addition, we also study the ultimate limits to sensitivity enhancements in such sensors imposed by the bandwidth of coupled dynamics.

\section{MODE-LOCALIZED SENSING: SENSITIVITY ANALYSIS}

Most mode-localized sensors rely on utilizing weakly coupled nearly identical arrays of mechanical resonators as platforms for mechanical sensing. In such an array of identical resonators coupled through weak springs, even a small perturbation in the structural properties of one of the coupled resonators inhibits the propagation of vibrations within the system leading to a confinement of vibration energy to small geometric regions. The extent of this vibration energy confinement depends not only on the magnitude of the periodicity breaking irregularity, but also on the strength of internal coupling between the resonators, with weaker coupling resulting in stronger vibration confinement. ${ }^{10,12}$ This phenomenon consequently results in a localization of the vibration modes under conditions of weak elastic coupling between the resonators, resulting in drastic variations of the eigenstates (the normalized eigenvectors/mode shapes) that may be as high as orders of magnitude greater than corresponding shifts in resonant frequency for the same induced structural perturbation.

In order to understand the underlying physics in more detail, consider two resonators coupled through a spring $\left(k_{c}\right)$ as represented in the discretized model shown in Fig. 1. The variation in the eigenstates due to an induced periodicitybreaking structural perturbation on one of the two coupled structures may be evaluated using the Rayleigh's Energy method. ${ }^{14}$ In the case of a perfectly periodic system wherein the two coupled resonators are mechanically identical (i.e., $k_{1}=k_{2}=k$ and $m_{1}=m_{2}=m$ ), the system is symmetric about the coupling spring and the mode shapes may simply be deduced by inspection to be symmetric and anti-symmetric at the two fundamental modes of vibration (corresponding to $x_{2} / x_{1}=1$ and $x_{2} / x_{1}=-1$ respectively where $x_{1}$ and $x_{2}$ represent the amplitudes of vibration of the resonators 1 and 2 , respectively, at the two fundamental modes of the coupled system).

The relative variation in the eigenstates of the discretized 2 degree of freedom (2 DOF) system due to an induced mass addition on one of the coupled resonators (say resonator 2) relative to the other (i.e., when $m_{2}=m\left(1+\delta_{m}\right)$, $\left.m_{1}=m\right)$, may be estimated to be

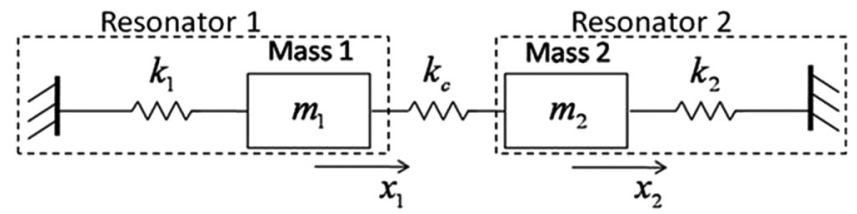

FIG. 1. Undamped two-degree of freedom (2 DOF) spring-mass system.

$$
\Delta\left|\frac{x_{2}}{x_{1}}\right| \approx \frac{\delta_{m}}{2 \kappa},
$$

where $\delta_{m}=\Delta m / m$ corresponds to the nondimensionalized mass perturbation on resonator 2 relative to resonator 1 and $\kappa=k_{c} / k$ represents the nondimensionalized (or scaled) coupling factor.

Similarly, evaluating for the variation in mode shape at the first eigenvalue for an induced perturbation in the stiffness of resonator 2 relative to resonator 1 (i.e., when $\left.k_{2}=k\left(1+\delta_{k}\right), k 1=k\right)$, we get

$$
\Delta\left|\frac{x_{2}}{x_{1}}\right| \approx\left|-\frac{\delta_{k}}{2 \kappa}\right|=\frac{\delta_{k}}{2 \kappa} .
$$

where $\delta_{k}=\Delta k / k$ corresponds to the nondimensionalized stiffness perturbation on resonator 2 relative to resonator 1 . From Eq. (1) and Eq. (2), the sensitivity of the system, which corresponds to the relative shift in the mode shape of the 2 DOF mode-localized sensor, may be expressed as:

$$
\Delta\left|\frac{x_{2}}{x_{1}}\right| /\left|\frac{x_{2}^{0}}{x_{1}^{0}}\right|=\frac{\delta}{2 \kappa}
$$

where $\delta=\delta_{m}$ or $\delta_{k}$ for the case of a mass or a stiffness perturbation on one of the coupled resonators, respectively; $x_{1}^{0}$ and $x_{2}^{0}$ represent the unperturbed, deterministic amplitudes of vibration of the two weakly coupled resonators 1 and 2 and $\left(x_{2}^{0} / x_{1}^{0}\right)$ the corresponding unperturbed mode shape. From Eq. (3), it may be observed that lowering the strength of internal coupling between the resonators, should enhance the output response of the system. This critical dependence of the system response of such mode-localized sensors on the strength of internal coupling has been exploited in recent years to attain output sensitivities that are as high as orders of magnitude greater than corresponding variations in resonant frequency for the same structural perturbation. ${ }^{8-12}$ It is to be noted here, that while these results hold true in the case of two weakly coupled undamped resonators, the undamped eigenmode character of the solution should be preserved even for the case of two damped resonators (as represented in Fig. 2), so long as the damping is proportional [i.e., the damping matrix of the system is simultaneously diagonalizable with the mass $(M)$ and stiffness $(K)$ matrices of the system as expressed in Eq. (4)].

$$
C=\alpha M+\beta K
$$

This is because, in the case of a proportionally damped system, substituting Eq. (4) into the equation of motion of an array of $n$-coupled resonators and using the principle of orthogonality would result in a set of $n$-completely

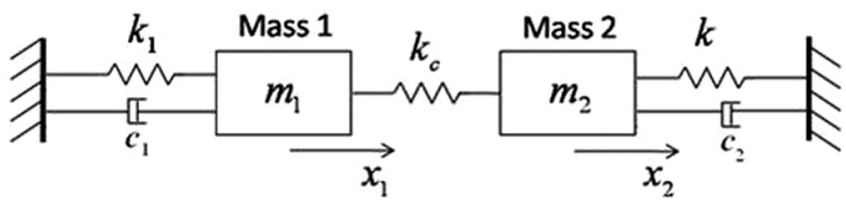

FIG. 2. Damped lumped element model of two coupled resonators. 
decoupled equations of motion, each governing the motion of one particular eigenvector (similar to the undamped case $\left.^{14}\right)$. Since there exists no coupling between the eigenvectors in such a case, the extracted mode shapes of a proportionally damped system would be identical to those computed for the undamped case but would have phase differences that govern the rate of decay of coupled oscillations [dictated by the parameters $\alpha, \beta$ and the resonant frequency $\omega$ (corresponding to that of the decoupled harmonic oscillator)]. This special form of damping is often referred to in structural vibration literature as Rayleigh damping and applies in the case of most micro- or nanomechanical mode-localized sensors that are comprised of coupled, nearly matched resonators operating in the same ambient environment. This firstorder independence of the mode shape to environmental drift becomes especially useful in the context of mode-localized sensing, as it leads to an important, inherent advantage of common mode rejection (CMR) in such sensors. This CMR behavior has been experimentally demonstrated elsewhere. ${ }^{11}$

However, an important question remains to be addressed: what is the minimum shift in the mode shape that may be resolved in a multi-DOF mode-localized sensor? Since the mode shapes are deduced from the relative amplitudes of vibration of the coupled resonators at the fundamental modes, the resolution of a mode-localized sensor should depend critically not only on the improved system response [given by Eq. (3)], but also on the ability to resolve even the smallest fluctuations in the modal amplitudes (i.e., the amplitudes at the fundamental modes) when subjected to a differential symmetry breaking structural perturbation. It is to be noted here that any variation in the modal amplitudes $\left(\Delta\left|x_{2} / x_{1}\right|\right)$ is resolvable only so long as the measured individual amplitudes of each of the coupled resonators is greater than the root mean square amplitude fluctuations induced by noise in the system. The minimum resolvable mode-shape variation can therefore be expressed as

$$
\Delta\left|\frac{x_{2}}{x_{1}}\right|_{\min } /\left|\frac{x_{2}^{0}}{x_{1}^{0}}\right|=\Delta\left(\left|\frac{x_{2}^{0} \pm x_{2}^{\text {noise }}}{x_{1}^{0} \pm x_{1}^{\text {noise }}}\right|-\left|\frac{x_{2}^{0}}{x_{1}^{0}}\right|\right) /\left|\frac{x_{2}^{0}}{x_{1}^{0}}\right|,
$$

$x_{2}^{\text {noise }}$ and $x_{1}^{\text {noise }}$ correspond to the modal amplitude fluctuations of resonators 2 and 1 (in the case of the 2 DOF modelocalized sensor represented in Fig. 2) induced by noise in the system. It can be shown that Eq. (5) can be simplified further and expressed as

$$
\Delta\left|\frac{x_{2}}{x_{1}}\right|_{\min } /\left|\frac{x_{2}^{0}}{x_{1}^{0}}\right|=\Delta\left(\frac{x_{2}^{\text {noise }}}{x_{2}^{0}} \pm \frac{x_{1}^{\text {noise }}}{x_{1}^{0}}\right) .
$$

If $X_{r}^{0}$ represents the deterministic amplitude of vibration of the $r$ th coupled harmonic oscillator at its fundamental mode and $\Delta x_{r}^{\text {noise }}$ represents the standard deviations in the modal amplitude response of the $r$ th coupled resonators caused by random noise, then the minimum resolvable shift in the mode shape may be evaluated from Eq. (6) as,

$$
\Delta\left|\frac{x_{2}}{x_{1}}\right|_{\min } /\left|\frac{x_{2}^{0}}{x_{1}^{0}}\right|=\sqrt{\sum_{r=1}^{2}\left(\frac{\left\langle\Delta x_{r}^{\text {noise }}\right\rangle}{X_{r}^{0}}\right)^{2}} .
$$

Equation (7) represents the resolution of a mode-localized sensor comprising of two nearly identical, weakly coupled micro- or nanomechanical resonators and would remain valid so long as the standard deviations in the modal amplitude response of each of the resonators are independent. From Eq. (7), it is clear that the resolution is dictated by the amplitude stability $\left(\left\langle\Delta x_{r}^{\text {noise }}\right\rangle\right)$ of each of the two coupled resonators at their fundamental modes of vibration. It is to be noted that while the description so far concerns the sensitivity of an array consisting of two weakly coupled micromechanical/nanomechanical resonators, a further increase in the number of coupled resonators could provide an additional improvement in the sensitivity/responsivity of the system. ${ }^{9,10}$ However increasing the number of resonators will correspondingly increase the injected noise as well, as the measured output would then require accurate quantifications of the relative amplitudes of vibration of all the coupled resonators at the fundamental modes as may be seen by extending Eq. (7).

As discussed earlier, any amplitude shift comparable to the mean square noise in an ensemble of amplitude variations is resolvable only so long as the signal-to-noise ratio $(S N R)$ satisfies the relation $S N R \geq 1$. An estimate of this minimum resolvable resonant amplitude shift may hence be obtained by integrating the effective spectral density function of the amplitude fluctuations induced by noise within a frequency band of $-\Delta \omega$ to $\Delta \omega$ (in the output frequency spectrum) that corresponds to a frequency range near the resonant frequency of interest:

$$
\left\langle x_{\min }\right\rangle \geq\left\langle\Delta x^{\mathrm{noise}}\right\rangle=\sqrt{\frac{1}{2 \pi} \int_{-\Delta \omega}^{\Delta \omega} S_{x x}(\omega) d \omega},
$$

where $x$ represents the fluctuation in resonant amplitude and $S_{x x}$, the power spectral density. While Eq. (8) is a general expression that denotes the root mean square amplitude fluctuations measured from the system, the precise form of the spectral density function $\left(S_{x x}\right)$ depends upon the noise processes that are operative in the system within the specified frequency band.

\section{MECHANICAL NOISE CONTRIBUTIONS}

\section{A. Thermomechanical noise fluctuations}

One of the fundamental factors that limits the resolution of a mode-localized sensor is thermomechanical noise. Thermomechanical or mechanical-thermal noise originates from thermally driven random motion of a mechanical system that serves as the platform for mode-localized sensing. It arises from the dynamic equilibrium between the mechanical energy of the device and the thermal energy of the surrounding ambient environment and usually has a broad-band white spectrum. ${ }^{15}$ In order to evaluate the impact of thermomechanical noise on the resolution of a weakly coupled multidegree of freedom (MDOF) mode-localized sensor, we first determine the frequency-response function of the system. 
The equation of motion of the MDOF sensor may be written in the form:

$$
M \ddot{x}+C \dot{x}+K x=F,
$$

where $M, C$, and $K$ represent the mass, damping and the stiffness matrices of the system, and $x$ and $F$ represent the vectors of displacements and force. A Fourier transform of this equation yields:

$$
\left[-\omega^{2} M+i \omega C+K\right] x(\omega)=F(\omega),
$$

where $F(\omega)$ and $x(\omega)$ represent the Fourier transforms of the force and response matrices. It then follows that

$$
x(\omega)=H(\omega) F(\omega) ; H(\omega)=\left[-\omega^{2} M+i \omega C+K\right]^{-1},
$$

where, $H(\omega)$ denotes the frequency response matrix of the sensor.

The general motion of any MDOF system may also be written as a linear combination of modal deformations ${ }^{16}$ as expressed in Eq. (12):

$$
\underline{x}=q_{1}(t) \underline{u_{1}}+q_{2}(t) \underline{u_{2}}+\ldots+q_{n}(t) \underline{u_{n}}=\sum_{r=1}^{n} q_{r}(t) \underline{u_{r}},
$$

where $u_{r}$ represents the $r$ th modal eigenvector of the dynamic system and $q_{r}(t)$, its corresponding normal coordinate. In the case where the coupled nearly identical resonators are proportionally damped, the matrices $M, K$, and $C$ of the system are symmetric and the eigenvectors of the system are orthogonal as explained in the previous section. Hence, each normal coordinate of the system should obey a simple single degree-of-freedom (1 DOF) harmonic resonator equation, independent of all others. ${ }^{16}$ Assuming that the drive force is purely thermal in origin, the $r$ th individual equation of motion of the system may then be re-expressed in the form:

$$
m_{r} \ddot{q}_{r}(t)+c_{r} \dot{q}_{r}(t)+k_{r} q_{r}(t)=f_{r}(t),
$$

where $m_{r}, c_{r}, k_{r}$ and $f_{r}(t)$, represent the mass, damping, stiffness and thermal forcing term of the $r$ th individual resonator.

From Eq. (13) and Eq. (12), it is clear that by evaluating the impact of thermomechanical noise on the $r$ th individual normal coordinate, one may be able to estimate the impact of thermomechanical noise on the net sensitivity of the MDOF mode-localized sensor. Thus, before proceeding with the thermal noise analysis of the MDOF mode-localized sensor, we first evaluate the input-output response of a single ( $r$ th) resonator to a stationary random thermal noise input.

Just as in Eq. (11), the response function of the $r$ th resonator may be expressed in the form,

$$
H_{r}(\omega)=\left[-\omega^{2} m_{r}+i \omega c_{r}+k_{r}\right]^{-1} .
$$

From Eq. (14), the spectral density function of the $r$ th harmonic oscillator for a stationary random thermal excitation may be evaluated using the Weiner-Khintchine excitationresponse relation as ${ }^{17}$

$$
S_{q_{r} q_{r}}(\omega)=\left|H_{r}(\omega)\right|^{2} S_{f_{r} f_{r}}(\omega),
$$

where $S_{f_{r} f_{r}}(\omega)$ is the input noise excitation power spectral density of the $r$ th harmonic oscillator in $f_{r}(t)$. Based on the Equipartition theorem, ${ }^{15}$ any mode of the system in thermal equilibrium has an average thermal noise energy given by $k_{B} T / 2$, where $k_{B}$ represents the Boltzmann's constant and $T$, the absolute temperature. Hence,

$$
\frac{1}{2} k_{B} T=\frac{1}{2} k_{r}\left\langle q_{r}(t)^{2}\right\rangle,
$$

where $\left\langle q_{r}(t)^{2}\right\rangle$ represents the mean square fluctuation of the general coordinate corresponding to the modal deformation on the $r$ th harmonic resonator. Equation (16) may be rewritten in terms of the auto-correlation function $R_{q_{r} q_{r}}(\tau)$ [that represents the ensemble of the product of $q_{r}(t)$ when sampled at time $t$ and that when sampled at $t+\tau$ within the limits $R_{q_{r} q_{r}}(0)=E\left[q_{r}(t)^{2}\right]$ and $R_{q_{r} q_{r}}(\infty)$ $\left.=\mu^{2}\left\{\mu=E\left[q_{r}(t)\right]\right\}^{17}\right]$ as:

$$
\frac{1}{2} k_{B} T=\frac{1}{2} k_{r}\left\langle q_{r}(t)^{2}\right\rangle=\frac{1}{2} k_{r} R_{q_{r} q_{r}}(0)=\frac{1}{4 \pi} k_{r} \int_{-\infty}^{\infty} S_{q_{r} q_{r}}(\omega) d \omega .
$$

Now, substituting Eqs. (14) and (15) into Eq. (17) enables the evaluation of the excitation spectral density: ${ }^{18}$

$$
\begin{aligned}
& \frac{1}{2} k_{B} T=\frac{1}{4 \pi} k_{r} \int_{-\infty}^{\infty}\left|\frac{1}{-\omega^{2} m_{r}+i \omega c_{r}+k_{r}}\right|^{2} S_{f_{r} f_{r}}(\omega) d \omega \\
& \Rightarrow \frac{1}{2} k_{B} T=\frac{S_{f_{r} f_{r}}(\omega)}{4 \pi} \int_{-\infty}^{\infty} \frac{k_{r}}{\left(k_{r}-\omega^{2} m_{r}\right)^{2}+\left(\omega c_{r}\right)^{2}} d \omega
\end{aligned}
$$

Evaluating the above integral when the damping factor is less than 1 (i.e., when the quality factor of resonance, $Q>1$ ) yields ${ }^{18}$

$$
\int_{-\infty}^{\infty} \frac{1}{\left(k_{r}-\omega^{2} m_{r}\right)^{2}+\left(\omega c_{r}\right)^{2}} d \omega \approx \frac{\pi}{\omega_{r}^{2} c_{r} m_{r}} .
$$

Substituting Eqs. (19) into (18) yields

$$
\frac{1}{2} k_{B} T=\frac{S_{f_{r} f_{r}}}{4 \pi} \frac{k_{r} \pi}{\omega_{r}^{2} c_{r} m_{r}} \Rightarrow S_{f_{r} f_{r}}=2 k_{B} T c_{r} .
$$

The mean square displacement of the $r$ th harmonic oscillator due to a thermomechanical spectral noise input within a frequency band of $-\Delta \omega$ to $\Delta \omega$ may now be evaluated as:

$$
\begin{aligned}
\left\langle\left(\Delta x_{r}^{n o i s e}\right)^{2}\right\rangle & =\frac{1}{2 \pi} \int_{-\Delta \omega}^{\Delta \omega} S_{q_{r} q_{r}}(\omega) d \omega \\
& =\int_{-\Delta f}^{\Delta f} \frac{2 k_{B} T \underline{u}_{r}^{2} c_{r}}{\left(k_{r}-\omega^{2} m_{r}\right)^{2}+\left(\omega c_{r}\right)^{2}} d \omega \approx \frac{4 k_{B} T \underline{u}_{r}^{2} c_{r} \Delta f}{m_{r}^{2} \omega_{r}^{4}},
\end{aligned}
$$


where $\Delta \omega \ll \omega_{r}$ and $\Delta f=\Delta \omega /(2 \pi)$. From Eq. (21), the minimum measurable displacement for the $r$ th individual oscillator of the coupled MDOF system can be derived as:

$$
\left\langle x_{r}^{\mathrm{min}}\right\rangle=\left\langle\Delta x_{r}^{\mathrm{noise}}\right\rangle=\sqrt{\left\langle\left(\Delta x_{r}^{\text {noise }}\right)^{2}\right\rangle}=\sqrt{\frac{4 k_{B} T c_{r}^{\text {eff }} \Delta f}{\left(m_{r}^{\text {eff }}\right)^{2}\left(\omega_{r}^{\text {eff }}\right)^{4}}},
$$

where $m_{r}^{\text {eff }}, c_{r}^{\text {eff }}$, and $\omega_{r}^{\text {eff }}$ denote the effective modal mass, damping and angular resonant frequency of the $r$ th individual oscillator.

Under conditions of low modal overlap (i.e., when the ratio of the half power bandwidth to the frequency spacing between adjacent modes is less than $1 \Rightarrow\left[2 \omega_{r} c_{r} / \mid \omega_{r+1}-\right.$ $\left.\omega_{r} \mid \ll 1\right]$ ), Eqs. (15) and (12) may be used to derive the net response spectral density function of the MDOF system for the random thermal excitation as:

$$
\begin{gathered}
S_{x x}(\omega)=\sum_{r=1}^{n} S_{q_{r} q_{r}}(\omega) \underline{u}_{r}{ }^{2} \\
S_{x x}(\omega)=\sum_{r=1}^{n} \frac{S_{0}}{\left(k_{r}-\omega^{2} m_{r}\right)^{2}+\left(\omega c_{r}\right)^{2}} \frac{u_{r}{ }^{2}}{2 k_{B} T \underline{u}_{r}{ }^{2}} c_{r} \\
=\sum_{r=1}^{n} \frac{\left.\omega^{2} m_{r}\right)^{2}+\left(\omega c_{r}\right)^{2}}{\left(k_{r}-\omega^{2}\right.}
\end{gathered}
$$

Equation (24) corresponds to the response spectral density function of the MDOF mode-localized sensor to a stationary random thermal noise input.

Since the response of the system relies on estimating the mode shapes of the system from the measured amplitudes of vibration of each of the coupled resonators at their fundamental modes, the amplitude fluctuations represented in Eq. (22) may directly be employed to determine the effective noise contribution in the modal response when measured from the $r$ th coupled harmonic resonator of the multi-degree of freedom sensor:

$$
\begin{aligned}
\frac{x_{r}^{\min }}{X_{r}^{0}} & =\frac{2 \sqrt{\frac{\left(k_{B} T c_{r} \Delta f\right)}{\left(m_{r}^{2} \omega_{r}^{4}\right)}}}{X_{r}^{0}}=2 \sqrt{\frac{k_{B} T c_{r}^{\text {eff }} \Delta f}{\left(m_{r}^{\text {eff }}\right)^{2}\left(\omega_{r}^{\text {eff }}\right)^{4}\left(X_{r}^{0}\right)^{2}}} \\
& =2 \sqrt{\frac{E_{t h} \Delta f}{E_{c} Q \omega_{r}^{\text {eff }}}}
\end{aligned}
$$

where $X_{r}^{0}$ denotes the maximum deterministic amplitude of the $r$ th coupled resonator at its fundamental mode of vibration, $E_{t h}=k_{B} T / 2$ represents the thermal energy on the $r$ th harmonic oscillator and $E_{c}$, its corresponding maximum drive energy given by Eq. (26).

$$
E_{c}=\left[m_{r}^{e f f}\left(\omega_{r}^{e f f}\right)^{2}\left\langle\left(X_{r}^{0}\right)^{2}\right\rangle\right] / 2
$$

The minimum resolution of the mode localized sensor comprising of two nearly identical resonators would then correspond to [from Eq. (7)]

$$
\Delta\left|\frac{x_{2}}{x_{1}}\right|_{\min }|| \frac{x_{2}^{0}}{x_{1}^{0}} \mid=\sqrt{\sum_{r=1}^{2}\left(\frac{\left\langle\Delta x_{r}^{\text {noise }}\right\rangle}{X_{r}^{0}}\right)^{2}}=4 \sqrt{\frac{E_{t h} \Delta f}{2 E_{c} Q \omega_{r}^{\text {eff }}}} .
$$

Substituting Eqs. (27) in (3), the minimum resolvable shift in mass/stiffness may be estimated as

$$
\frac{\delta}{2 \kappa} \approx 4 \sqrt{\frac{E_{t h} \Delta f}{2 E_{c} Q \omega_{r}^{\mathrm{eff}}}} .
$$

Assuming that the two resonators are initially identical, Eq. (28) may be rewritten for a mass perturbation on one of the two nearly identical resonators as

$$
\frac{\Delta m_{\mathrm{min}}}{m_{\mathrm{eff}}} \approx 8 \kappa \sqrt{\frac{E_{t h} \Delta f}{2 E_{c} Q \omega_{\mathrm{eff}}}},
$$

where $m_{\text {eff }}$ and $\omega_{\text {eff }}$ denotes the effective modal mass and angular resonant frequency of each of the weakly coupled identical harmonic resonators (before an induced mass perturbation). The minimum resolvable shift in stiffness may be evaluated in a similar fashion to get:

$$
\frac{\Delta k_{\mathrm{min}}}{k_{\mathrm{eff}}} \approx 8 \kappa \sqrt{\frac{E_{t h} \Delta f}{2 E_{c} Q \omega_{\mathrm{eff}}}} .
$$

Equations (29) and (30) represent the fundamental resolution of a 2 DOF mode-localized sensor induced by thermomechanical noise when subjected to induced differential perturbations in mass and stiffness, respectively, when measured from the first fundamental mode of vibration. Comparing this resolution with those attainable in the conventional resonant frequency shift based sensing scheme [the mass resolution of which is expressed in Eq. $(31)^{19}$ ], we notice that the resolution in this paradigm of mechanical sensing scales proportionally with the scaled coupling factor " $k$ " as elucidated by Eq. (32).

$$
\begin{gathered}
\frac{\Delta m_{\mathrm{min}}}{m_{\mathrm{eff}}} \approx 2 \sqrt{\frac{E_{t h} \Delta f}{E_{c} Q \omega_{\mathrm{eff}}}} \\
\frac{\left(\Delta m_{\mathrm{min}}\right)_{\mathrm{mod}}}{\left(\Delta m_{\mathrm{min}}\right)_{\mathrm{freq}}} \propto \kappa .
\end{gathered}
$$

\section{B. Momentum-exchange noise}

Next, we evaluate the limits imposed due to the momentumexchange between the micro- or nanomechanical resonators (that form a part of the weakly coupled mode-localized sensor array) and the gas molecules from the surrounding medium that impinge upon them. The complement of this noise process (thermomechanical noise) was considered previously.

In the molecular region, the equation of motion of the $r$ th individual harmonic resonator when subjected to viscous drag takes the form: ${ }^{20}$

$$
\left(m_{r}\right) \ddot{x}_{r}(t)+F_{r \mathrm{drag}}+k_{r} x_{r}(t)=f_{r}(t),
$$

where the drag force, $F_{r \text { drag }}$ consists of two components: ${ }^{20}$ one resulting from the intrinsic damping within the resonator and another, resulting from the interaction between the resonator and the gas molecules of the ambient viscous environment. 
The drag force may hence, be represented in the following form $^{20}$

$$
F_{r_{\text {drag }}}=\left(c_{r_{\text {drag }}}^{\text {oscillator }}\right) \dot{x}_{r}+\left(c_{r_{\text {drag }}}^{\text {ambient }}\right) \dot{x}_{r}+\left(c_{r_{\text {drag }}}^{\text {inertial }}\right) \ddot{x}_{r} .
$$

Substituting Eqs. (34) into (33), we get

$$
\begin{aligned}
& \left(m_{r}+c_{r_{\text {drag }}}^{\text {inertial }}\right) \ddot{x}_{r}(t)+\left(c_{r_{\text {drag }}}^{\text {ambient }}+c_{r_{\text {drag }}}^{\text {oscillator }}\right) \dot{x}_{r} \\
& +k_{r} x_{r}(t)=f_{r}(t) .
\end{aligned}
$$

It may be observed that the drag force consists of both inertial components that are proportional to the acceleration and dissipative components. Depending on the pressure of the ambient and the intrinsic quality factor of the harmonic oscillator, the impact of the inertial and dissipative components of the drag force on the dynamic behavior would vary. ${ }^{20}$ The inertial component of the drag force (that is the component of $F_{r \text { drag }}$ proportional to the acceleration) has an effect of increasing the mass of the vibrating oscillator (from $m_{r}$ to $m_{r}+c_{r_{\text {drag }}}^{\text {inertial }}$ ), the influence of which becomes negligible at lower pressure conditions. Hence, when such sensors are operated in vacuum, the drag force should simply consist of the component describing the intrinsic damping within the oscillator $\left(c_{r_{\text {drag }}}^{\text {oscillator }}\right)$ and the dissipative drag proportional to the velocity of oscillation ( $\left.c_{r_{\text {drag }}}^{\text {ambient }}\right)$ as elaborated by Kokubun et $a .^{20}$ The damped equation of motion of the $r$ th harmonic resonator under conditions of low ambient pressures may thus be rewritten as:

$$
\left(m_{r}\right) \ddot{x}_{r}(t)+\left(c_{r_{\text {drag }}}^{\text {ambient }}+c_{r_{\text {drag }}}^{\text {oscillator }}\right) \dot{x}_{r}+k_{r} x_{r}(t)=f_{r}(t) .
$$

From Eq. (36), it is clear that there exists a simple superposition of damping mechanisms under low ambient pressure conditions and hence, the net quality factor at the fundamental mode of the $r$ th harmonic resonator may be expressed as:

$$
\frac{1}{Q_{r}}=\sum_{n} \frac{1}{Q_{r}^{n}}=\frac{1}{Q_{r}^{\text {oscillator }}}+\frac{1}{Q_{r}^{\text {ambient }}} .
$$

From Eq. (37), it is obvious that the magnitude of $Q_{r}$ cannot exceed the value of the smallest individual quality factor arising from each of the damping mechanisms and thus, instead of estimating the quality factors of each individual damping mechanism, it would suffice to approximate the expression by considering the damping component of the drag force that dominates in that region. In the case where in the intrinsic damping of the resonator is much lower than that arising from the ambient, i.e., when $Q_{r}^{\text {oscillator }} \gg Q_{r}^{\text {ambient }}$, the quality factor of the $r$ th harmonic oscillator would thus correspond approximately to that arising from collisions with surrounding environmental gas molecules alone and hence, from Eq. (37), the input spectral density of momentum exchange noise may be expressed in the form

$$
S_{M E}=2 k_{B} T c_{r_{\text {drag }}}^{\text {ambient }} .
$$

The response of the system may now be derived using the Weiner-Khintchine input output response, in a similar manner to that estimated previously for thermomechanical noise, resulting in:

$$
S_{x x}(\omega)=\sum_{r=1}^{n} \frac{2 k_{B} T \underline{u_{r}}{ }^{2} c_{r_{\text {drag }}^{\text {ambient }}}}{\left(k_{r}-\omega^{2} m_{r}\right)^{2}+\left(\omega c_{r_{\text {drag }}^{\text {ambient }}}\right)^{2}} .
$$

From Eq. (39), the minimum resolvable shifts in mass and stiffness due to momentum-exchange may be evaluated as:

$$
\begin{aligned}
\frac{\Delta m_{\mathrm{min}}}{m_{\mathrm{eff}}} & \approx 8 \kappa \sqrt{\frac{E_{t h} c_{r_{\text {drag }}}^{\text {ambient }} \Delta f}{2 E_{c} m_{r} \omega_{r}^{2}}} \\
\frac{\Delta k_{\mathrm{min}}}{k_{\mathrm{eff}}} & \approx 8 \kappa \sqrt{\frac{E_{t h} c_{r_{\text {drag }}}^{\text {ambient }} \Delta f}{2 E_{c} m_{r} \omega_{r}^{2}}} .
\end{aligned}
$$

It is to be noted that the above derivation is valid only when the micromechanical resonator arrays are operated in partial/ high vacuum environments, as at higher ambient pressures the effect of the inertial drag term would also have to be considered in the analysis.

\section{BANDWIDTH LIMIT}

Although it is clear from Eq. (32) and Eq. (40) that lowering the effective normalized coupling spring constant should result in better resolutions in this sensing paradigm when compared to those attainable using the traditional resonant frequency shift based sensing approach, it is to be noted that reducing " $k$ " would also significantly alter the coupled dynamics of the system by lowering the frequency separation between the two modes. ${ }^{21}$ This consequently imposes a practical bandwidth limit on the minimum usable coupling $\kappa$ and in effect, the minimum attainable resolution in this sensing paradigm. This is because in order to obtain a simple estimate of the eigenstate variations in such 2 DOF sensors as derived in the previous section, the frequency separation between the two resonant peaks would have to be larger than the halfwidth of each of the resonances. This becomes a mandatory condition that needs to be satisfied for the single-mode approximation made in the previous analysis to hold true.

Under conditions of weak internal coupling, the frequencies corresponding to the in-phase and anti-phase modes may be written as:

$$
\omega_{1}^{2}=\frac{k}{m} ; \quad \omega_{2}^{2}=\frac{\left(k+2 k_{c}\right)}{m} .
$$

If $\omega_{0}^{2}=k / m$, the frequency separation between the two modes may be derived as:

$$
\omega_{2}^{2}-\omega_{1}^{2}=\frac{2 k_{c}}{m}=2 \kappa \omega_{0}^{2} .
$$

Under conditions of weak internal coupling, the two eigenvalues are spaced close to each other [from Eq. (42)], and hence,

$$
\omega_{2}-\omega_{1} \approx \kappa \omega_{0}
$$

As mentioned earlier, the frequency separation between the two peaks would have to be larger than the half width of the 
resonances $\omega_{0} / Q$, and hence, the minimum practical value of $\kappa$ would be limited to

$$
\kappa_{\min } \geq \frac{1}{Q} .
$$

Substituting Eqs. (44) into (32), it is clear that the minimum resolvable shift in mass relative to the frequency based sensing would then be dictated by the quality factor of resonance at the fundamental mode of vibration as expressed in Eq. (45):

$$
\frac{\left(\Delta m_{\min }\right)_{\bmod }}{\left(\Delta m_{\min }\right)_{\text {freq }}} \propto \frac{1}{Q} .
$$

This is a very interesting result as it directly elucidates the dependence of the resolution in this sensing paradigm on the quality factor $(Q)$. This bandwidth trade-off should consequently dictate the ultimate limit to the minimum usable coupling strength that may be realized for operation in modelocalized resonator arrays.

\section{ELECTRONIC PREAMPLIFIER NOISE}

While the noise due to thermomechanical motion and momentum exchange dictate the ultimate resolution attainable in this sensing paradigm, in most practical applications, the noise fluctuation originating from the sensor's interfacial electronic preamplifier circuits tends to dominate the effective noise performance. In this section, we discuss and evaluate the impact of noise fluctuations arising from such electronic preamplifier circuits on the fundamental resolution of mode-localized sensors. However, unlike the previous analyses in the prior sections, we discuss the impact of preamplifier noise for the specific case wherein the micro- or nanomechanical resonator arrays are transduced electrically. The case of electrical/capacitive transduction was chosen for this particular analysis because of the ease of electromechanical transduction and for the purpose of experimental verification.

In most cases, the open-loop measurement circuit of an electrically transduced mode-localized sensor consists of a standard vector network analyzer (VNA) and a preamplifier circuit (used to amplify the motional current of each of the resonators) as illustrated in Fig. 3.

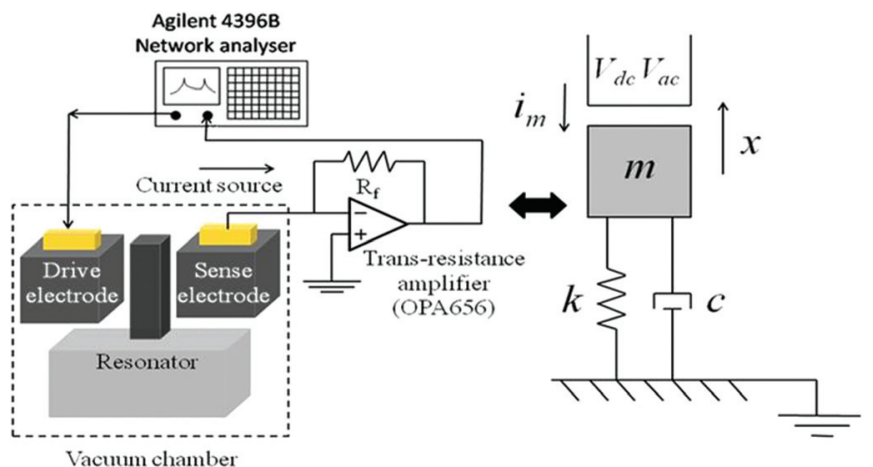

FIG. 3. (Color online) Conventional open-loop measurement setup in electrically transduced mechanical resonators.
A measurement of the scattering (S21) parameter response on the network analyzer allows for a proportional measure of the voltage transmission gain due to the motion of each of the coupled resonators as a function of the drive frequency (i.e., a measure of $\left(\vec{V}_{\text {out }} / \vec{V}_{\text {ac }}\right)$ where $\vec{V}_{\text {out }}$ represents the voltage output due to the motion of the resonator for an input RF voltage stimulus, $\vec{V}_{a c}$ ). If the preamplifier employed is a trans-resistance amplifier as illustrated in Fig. 3 , the measured S21 transmission should correspond to an amplified (but proportional) measure of the admittance transfer-function $\left(\vec{i}_{\text {out }} / \vec{V}_{\text {ac }}\right)$ of each of the coupled resonators. In order to evaluate the impact of noise on the measured admittance transfer-function, let us first evaluate the impact of noise on the current output $\vec{i}_{\text {out }}$ generated due to the motion of the resonators in the presence of a deterministic RF input voltage stimulus, $\vec{V}_{a c}$, from the network analyzer. Since the capacitive transduction scheme relies on measuring the motional current of each of the coupled resonators and in consequence, the admittance transfer function, each resonator in the coupled array of a mode-localized sensor may be modeled as an equivalent current source for the purpose of noise analysis.

Just as in the previous analyses, let us consider the case of a 2DOF mode-localized sensor similar to the one represented in Fig. 2. Under conditions of low modal overlap, the mechanical equation of motion of the $r$ th coupled resonator subjected to a harmonic electrical excitation may be expressed as in Eq. (13):

$$
m_{r} \ddot{q}_{r}(t)+c_{r} \dot{q}_{r}(t)+k_{r} q_{r}(t)=f_{r}(t) .
$$

In case of parallel plate capacitive transduction, the voltage applied across the drive capacitor results in a stored electrical potential energy, the derivative of which yields the force of actuation. An application of a dc and ac voltage across the capacitive drive electrode gap with $V_{d c} \gg\left|\vec{V}_{a c}\right|$, hence results in a drive force of magnitude: ${ }^{22}$

$$
\left.\overline{f_{r}} \approx\left(V_{d c} \frac{\partial C}{\partial x} \vec{V}_{a c}\right)\right|_{r}
$$

The output motional current from the $r$ th coupled resonator due to the drive force $f_{r}$ is given by the derivative of charge with respect to time

$$
i_{r}=\frac{d Q_{r}}{d t}=\left(C_{0}+d C\right) \frac{d V_{a c}}{d t}+\left(V_{d c}+V_{a c}\right) \frac{d C}{d t} .
$$

Rewriting Eq. (48) in phasor form,

$$
\overrightarrow{i_{r}}=\left(C_{0 j} j \omega_{r} \vec{V}_{a c}\right)+\left(\left.V_{d c} \frac{\partial C}{\partial x}\right|_{0} j \omega_{r} \vec{x}_{r}\right)+\left(\left.2 \vec{V}_{a c} \frac{\partial C}{\partial x}\right|_{0} j \omega_{r} \vec{x}_{r}\right) .
$$

If the applied $V_{d c}$ is much larger than $V_{a c}$, the current may be approximated as:

$$
\overrightarrow{i_{r}} \approx\left(C_{0} j \omega_{r} \vec{V}_{a c}\right)+\left(\left.V_{d c} \frac{\partial C}{\partial x}\right|_{0} j \omega_{r} \vec{x}_{r}\right)=\overrightarrow{i_{r}^{p a r a s i t i c}}+\overrightarrow{I_{r}^{0}},
$$


where $\overrightarrow{i_{r}^{p a r a s i t i c}}=\left(C_{0} j \omega_{r} \vec{V}_{a c}\right)$ represents the parasitic capacitive feedthrough current and $\overrightarrow{I_{r}^{0}}=\left.V_{d c} \frac{\partial C}{\partial x}\right|_{0} j \omega_{r} \vec{x}_{r}$ denotes the motional current originating from the motion of the $r$ th resonator. The equivalent electrical impedance (or the motional impedance) of the device hence corresponds to:

$$
Z_{m}=\frac{\vec{V}_{a c}}{\overrightarrow{I_{r}^{0}}}
$$

Since the mechanical impedance of the vibratory element is defined as the ratio of the force to the velocity, the admittance transfer function of a capacitively transduced resonator (after negating the effect of feedthrough parasitic ${ }^{22}$ ) may be derived using Eqs. (46) and (51) as:

$$
Y_{m}=\frac{\overrightarrow{I_{r}^{0}}}{\vec{V}_{a c}}=\left(\frac{\eta_{r}^{2} j \omega \vec{x}_{r}}{\bar{f}_{r}}\right)=\frac{\eta_{r}^{2}}{m_{r e q}}\left(\frac{\omega_{r}^{2}}{j \omega}+\frac{\omega_{0}}{Q_{r}}+j \omega\right)^{-1},
$$

where $\eta_{r}=\left.V_{d c} \frac{\partial C}{\partial x}\right|_{0}$ denotes the electromechanical transduction coefficient of the $r$ th individual resonator; $\omega_{r}$, its fundamental angular frequency of vibration; $m_{\text {req }}$, the equivalent mass of the resonator at the $r$ th fundamental natural frequency. Since the modal response of the system is evaluated by measuring the relative admittance transfer function responses of the two coupled resonators, the response of the sensor may be written as:

$$
\left|\frac{\left(Y_{m}\right)_{R 2}}{\left(Y_{m}\right)_{R 1}}\right|=\left|\left(\frac{\overrightarrow{I_{2}^{0}}}{\overrightarrow{V_{a c}^{R 2}}}\right)\right| /\left|\left(\frac{\overrightarrow{I_{1}^{0}}}{\overrightarrow{V_{a c}^{R 1}}}\right)\right| .
$$

Now, if the same deterministic RF input voltage stimulus $\left(\vec{V}_{a c}\right)$ is applied on both resonators simultaneously in such a way that $\overline{V_{a c}^{R 2}}=\overline{V_{a c}^{R 1}}$, then the minimum resolvable shift in the mode shape would simply correspond to the minimum measurable ratio of the motional currents of the two coupled resonators as expressed in Eq. (54):

$$
\Delta\left|\frac{\left(Y_{m}\right)_{R 2}}{\left(Y_{m}\right)_{R 1}}\right|_{\min } /\left|\frac{\left(Y_{m}\right)_{R 2}}{\left(Y_{m}\right)_{R 1}}\right|=\Delta\left|\frac{I_{2}}{I_{1}}\right|_{\min } /\left|\frac{I_{2}^{0}}{I_{1}^{0}}\right|=\Delta\left|\frac{x_{2}}{x_{1}}\right|_{\min } /\left|\frac{x_{2}^{0}}{x_{1}^{0}}\right|,
$$

where $\Delta\left|\frac{I_{2}}{I_{1}}\right|_{\min }|| \frac{I_{2}^{0}}{I_{1}^{0}}\left|=\Delta\left(\left|\frac{I_{2}^{0} \pm i_{2}^{\text {noise }}}{I_{1}^{0} \pm i_{1}^{\text {noise }}}\right|-\left|\frac{I_{2}^{0}}{I_{1}^{0}}\right|\right) /\right| I_{2}^{0}\left|\frac{I_{1}^{0}}{I_{1}^{0}}\right|$. From Eq. (54), it is clear that in order to evaluate the impact of the electronic preamplifier noise on the minimum measurable mode shape of the system, we may re-express all noise components as equivalent currents and simply evaluate the effect of noise on the measured motional current. Evaluating the preamplifier noise when modeling the $r$ th resonator as a current source results in Eq. (55):

$$
i_{r}^{\text {noise }}=\sqrt{\left(i_{r}^{\text {noise }}\right)^{2}}=\sqrt{\left[i_{n}^{2}+e_{n}^{2}\left(1+\frac{R_{s}}{R_{f}}\right)^{2} / R_{s}^{2}+\frac{4 k_{B} T}{R_{f}}\right]},
$$

where $i_{n}$ and $e_{n}$ represent the input current and voltage noise of the trans-resistance amplifier considered for the analysis (the values of which may be obtained from the datasheet of the amplifier chosen). $R_{s}$ and $R_{f}$ represent the motional resistance of the resonator at the fundamental resonant mode of interest and the amplifier feedback resistance, respectively. The minimum resolvable shift in the mode shapes of the 2 DOF mode-localized sensor when transduced electrically in open loop may hence be derived in a similar fashion to that of Eq. (7) to get:

$$
\begin{gathered}
\Delta\left|\frac{\left(Y_{m}\right)_{R 2}}{\left(Y_{m}\right)_{R 1}}\right|_{\min }|| \frac{\left(Y_{m}\right)_{R 2}}{\left(Y_{m}\right)_{R 1}} \mid \approx \Delta\left(\frac{i_{2}^{\text {noise }}}{I_{2}^{0}} \pm \frac{i_{1}^{\text {noise }}}{I_{1}^{0}}\right) \\
\Delta\left|\frac{\left(Y_{m}\right)_{R 2}}{\left(Y_{m}\right)_{R 1}}\right|_{\min } /\left|\frac{\left(Y_{m}\right)_{R 2}}{\left(Y_{m}\right)_{R 1}}\right| \approx \sqrt{\sum_{r=1}^{2}\left(\frac{\left\langle\Delta i_{r}^{\text {noise }}\right\rangle}{I_{r}^{0}}\right)^{2}},
\end{gathered}
$$

where $I_{r}^{0}$ represents the noiseless deterministic motional current of the $r$ th coupled resonator and $\Delta i_{r}^{\text {noise }}$, the noise currents of the $r$ th coupled resonator when characterized in open loop.

\section{EXPERIMENTAL METHODS}

In order to evaluate the noise performance of modelocalized sensors experimentally, we consider a mode-localized sensor comprising of a pair of electrically coupled, nearly identical microelectromechanical flexural wine glass mode ring resonators (an optical micrograph of which is shown in Fig. 4).

The device was fabricated in a commercial foundry process using the silicon-on-insulator microelectromechanical systems (SOI-MEMS) process through MEMSCAP Inc., USA. Each of the rings had an inner and outer diameter of $190 \mu \mathrm{m}$ and $220 \mu \mathrm{m}$, respectively, with a thickness of 10 $\mu \mathrm{m}$. Actuation was achieved using parallel plates of equal dimensions (120 $\mu \mathrm{m}$ long, $6 \mu \mathrm{m}$ wide, $10 \mu \mathrm{m}$ thick) attached to the anti-nodal points of the rings as shown in Fig. 4. The fabricated devices were tested under partial vacuum $(\approx 10$ mTorr) in a custom vacuum chamber. Figure 5 shows a schematic of the experimental measurement setup. All features including the drive and coupling gaps were designed to be 2 $\mu \mathrm{m}$ wide for both of the coupled ring resonators.

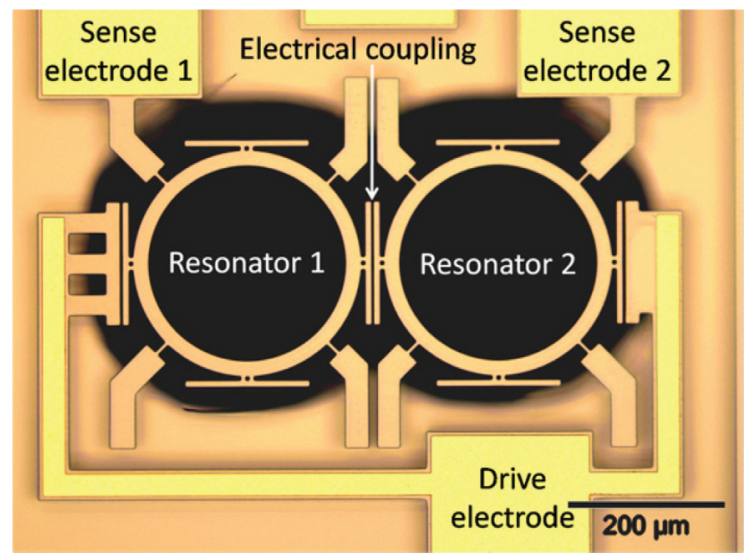

FIG. 4. (Color online) Optical micrograph of electrically coupled flexural wine-glass mode ring resonators. 


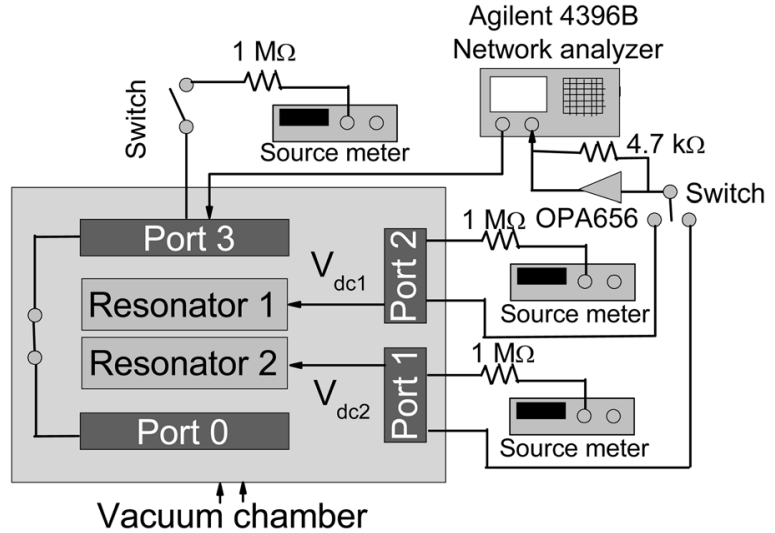

FIG. 5. Schematic of the measurement setup used for the characterization of electrically coupled resonator pairs.

Initially, DC polarization voltages of $+15 \mathrm{~V}$ and $-15 \mathrm{~V}$ were applied on resonators 1 and 2 through their respective sense electrodes (ports) while applying an ac drive power of $-15 \mathrm{dBm}$ on the drive electrode. The potential difference between the resonators should correspondingly result in the formation of an imaginary electro-elastic coupling spring across the coupling gap between the two resonators. ${ }^{10,19}$ This coupling behavior was verified experimentally by measuring the scattering (S21) parameter frequency responses as illustrated in Fig. 6 (extracted after parasitic capacitance cancellation using the procedure detailed by Yan et al. ${ }^{23}$ ). The quality factor at the first fundamental mode was measured to be approximately 13000 at a pressure of approximately $5 \mathrm{mTorr}$.

Before experimentally quantifying the fluctuation in the measured motional current of each of the coupled ring resonators, the expected variations in the current arising from the intrinsic and extrinsic noise sources discussed in the previous sections were theoretically evaluated as elaborated in Table I (the input noise current and noise voltage for the amplifier chosen (OPA656) was obtained from the datasheet and corresponded to $i_{n} \approx 1.3 f \mathrm{fA} / \sqrt{\mathrm{Hz}}$ and $e_{n} \approx 7 \mathrm{nV} / \sqrt{\mathrm{Hz}}$, respectively). The dominant noise component is boxed. (The impact of thermomechanical noise on the measured motional current is evaluated by first estimating the displacement due to thermal noise and then quantifying its corresponding current fluctuation as detailed in Table I).

It is clear that the fundamental noise floor imposed by the thermomechanical noise on the measured current noise is significantly lower in comparison to that induced by the associ-
TABLE I. Theoretical evaluation of current noise arising from various noise sources.

\begin{tabular}{|c|c|c|}
\hline Noise considered & $\begin{array}{l}\text { Effective noise } \\
\text { current }\left(A^{2} / \sqrt{H z}\right)\end{array}$ & Formula \\
\hline $\begin{array}{l}\text { Feedback thermal } \\
\text { resistance }\end{array}$ & $3.5 \times 10^{-24}$ & $\frac{4 k_{B} T}{R_{f}}$ \\
\hline Amplifier voltage noise & $2.2 \times 10^{-24} \quad e$ & $e_{n}^{2}\left(1+\frac{R_{s}}{R_{f}}\right)^{2} / R_{s}^{2} ;\left[R_{s}=\frac{m_{\text {eff }} \omega}{Q \eta^{2}}\right.$ \\
\hline Amplifier current noise & $1.7 \times 10^{-30}$ & $i_{n}^{2}$ \\
\hline Mechanical thermal noise & $4.32 \times 10^{-29}$ & $\left.i_{m}^{2} \approx\left|V_{d c} \frac{\partial C}{\partial x}\right|_{0} j \omega \vec{x}\right|^{2}$ \\
\hline
\end{tabular}

ated electronic interfacial circuitry (the amplifier voltage noise and the noise due to the feedback thermal resistance). The electronic noise should hence, dominate the effective noise performance of the sensor in this particular case. Calculating the net current noise in the system from Eq. (55), we get:

$$
\left\langle\Delta i_{\text {noise }}\right\rangle \approx 2.38 p A / \sqrt{H z} .
$$

Thus, for a measurement bandwidth of $10 \mathrm{~Hz}$, the minimum resolvable shift in the measured modal response of the $r$ th coupled resonator corresponds to

$$
\frac{\left\langle\Delta x_{r}\right\rangle}{X_{r}^{0}}=\frac{\left\langle\Delta i_{\text {noise }}\right\rangle}{I_{r}^{0}} \approx 0.0048 .
$$

From Eq. (59) and (57), the effective resolution of the sensor when the two coupled resonators are initially identical should relate to

$$
\Delta\left|\frac{\left(Y_{m}\right)_{R 2}}{\left(Y_{m}\right)_{R 1}}\right|_{\min }|| \frac{\left(Y_{m}\right)_{R 2}}{\left(Y_{m}\right)_{R 1}} \mid \approx \sqrt{\sum_{r=1}^{2}\left(\frac{\left\langle\Delta i_{r}^{\text {noise }}\right\rangle}{I_{r}^{0}}\right)^{2}} \approx 0.006 .
$$

This result was verified experimentally by measuring the mean and standard deviation of the modal amplitudes of motional current from the measured admittance spectra of resonators 1 and 2 at the first fundamental mode of vibration. This was done by studying the fluctuations in the output due to noise in the system for a set of samples (while maintaining the measurement parameters the same in both ring

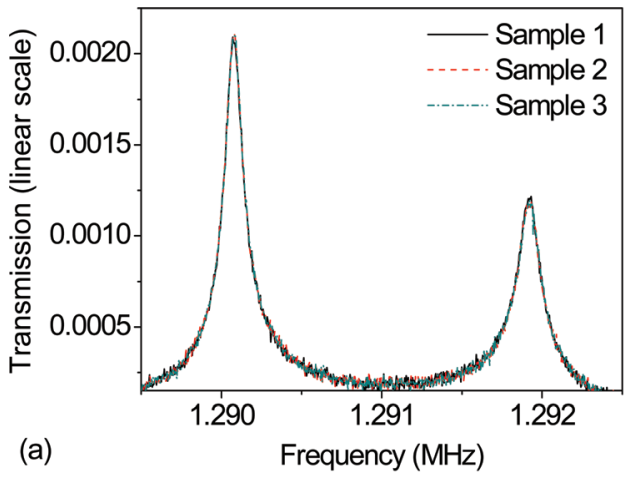

(b)

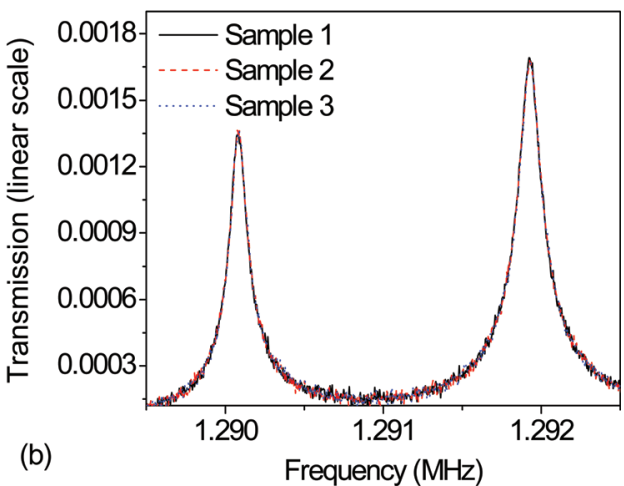

FIG. 6. (Color online) Schematic depicting the experimentally measured transmission responses of resonators 1 and 2 for a set of three samples while maintaining a constant drive input. 

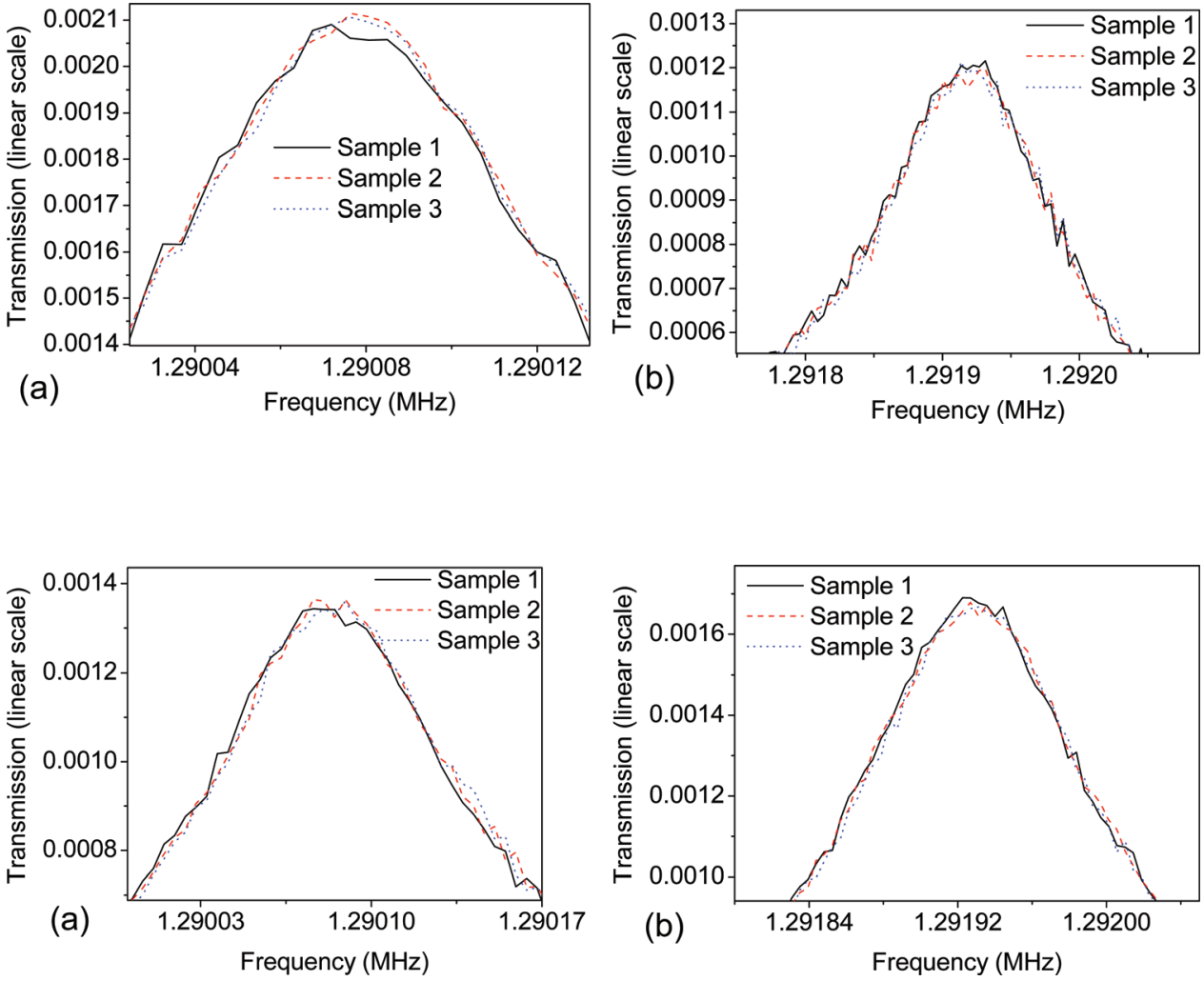

FIG. 7. (Color online) Measured transmission responses of resonator 1 at the (a) first and (b) second eigenvalues for a set of three samples while maintaining a constant drive input.

FIG. 8. (Color online) Measured transmission responses of resonator 2 at the (a) first and (b) second eigenvalues for a set of three samples while maintaining a constant drive input. resonators) and measuring the variations in the modal amplitude at the first eigenvalue from both resonators. The measured responses from the two coupled resonators are illustrated in Figs. 7 and 8. The measured responses of resonator 1 for a set of six samples are further elaborated in Table II.

Evaluating the minimum resolvable shifts in the mode shape from the results in Figs. 7 and 8 , we get $\left\langle\Delta i_{R 1}\right\rangle /$ $I_{R 1}^{0} \approx 0.0062$ from resonator 1 , and $\left\langle\Delta i_{R 2}\right\rangle / I_{R 2}^{0} \approx 0.0078$ from resonator 2 where $\Delta i_{R 1}$ and $\Delta i_{R 2}$ relate to the measured standard deviations in current measured from resonators 1 and 2, respectively, while $I_{R 1}^{0}$ and $I_{R 2}^{0}$ refer to their deterministic motional currents at the first fundamental mode of the

TABLE II. Measured fluctuation in the modal amplitudes measured from resonator 1 at the first and second fundamental modes of vibration.

\begin{tabular}{lcc}
\hline \hline Sample & $\begin{array}{c}\text { Amplitude measured at } \\
\text { first eigenvalue }\end{array}$ & $\begin{array}{c}\text { Amplitude measured at } \\
\text { second eigenvalue }\end{array}$ \\
\hline 1 & 0.002077175 & 0.001194426 \\
2 & 0.002090454 & 0.001215476 \\
3 & 0.002114550 & 0.001197169 \\
4 & 0.002106751 & 0.001213621 \\
5 & 0.002097941 & 0.001194710 \\
6 & 0.002100198 & 0.001202473 \\
Sum & 0.012587069 & 0.007217875 \\
Sample mean & 0.002097845 & 0.001202979 \\
Sample variance & $1.69164 \times 10^{-10}$ & $8.89919 \times 10^{-11}$ \\
Standard deviation of & $1.30063 \times 10^{-5}$ & $9.43355 \times 10^{-6}$ \\
$\quad$ measured current & & \\
\hline \hline
\end{tabular}

coupled vibratory behavior. This results in an effective noise floor of [from Eq. (60)]:

$$
\sqrt{\sum_{r=1}^{2}\left(\frac{\left\langle\Delta i_{r}^{\text {noise }}\right\rangle}{I_{r}^{0}}\right)^{2}} \approx 0.01 .
$$

The following conclusions may be made from the analysis: comparing the result derived theoretically [Eq. (60)] with that obtained from experiments [Eq. (61)], it is clear that the sensor noise floor measured experimentally is nearly consistent with predictions. While the sample size considered in this analysis corresponds to a set of six samples, larger sample sizes should yield a more accurate estimate of the statistical deviation that may be expected within the system. Nonetheless, it is evident that the dominant noise floor in this particular case is from the electronic interfacial preamplifier circuitry as elucidated analytically. Further optimization of the interface circuit should hence, help minimize this electronic noise floor to improve the practical resolution of the mode-localized sensor considered in this study. Alternative measurement schemes ${ }^{13,20}$ may also be adopted to obtain experimental estimates of the fundamental limits imposed by thermomechanical and momentum-exchange noise in such sensors.

\section{CONCLUSIONS}

In this paper, we evaluate the fundamental and practical limits to mode-localized sensing imposed by several important intrinsic and extrinsic noise sources, and the coupled 
dynamics. Our investigation of the fundamental limit on the performance of such mode-localized sensors results in the expression:

$$
\delta_{\min }=8 \kappa \sum_{r=1}^{n} \sqrt{\frac{E_{t h} \Delta f}{2 E_{c} Q \omega_{r}^{e f f}}},
$$

which makes transparent the most essential considerations for optimizing the ultimate sensitivity of mode-localized sensors. It is clear from Eq. (63), that one important parameter that distinguishes mode-localized sensors from the more conventional resonant frequency shift based sensing approach, is the resolution dependence on the strength of internal coupling, $\kappa$, between the coupled resonator arrays. Weaker effective coupling (lower $\kappa$ ) between the resonators should help enhance not only the responsivity of such sensors to an induced structural perturbation as elucidated in prior studies, but should also contribute to substantial improvements in the resolution under conditions of weak internal coupling. With recent reports detailing the realization of scaled (nondimensionalized) coupling factors, $\kappa \ll 10^{-3}$, improvements in the resolution by 3 to 4 orders of magnitude relative to corresponding resonant sensors should be possible using this unique sensing technique. Such resolution enhancements open the door to a new paradigm of mechanical sensing with ultimate sensitivity limits that are orders of magnitude greater than conventional resonant sensors. However, it is to be noted that reduction in the coupling factor significantly impacts the coupled dynamics by lowering the frequency separation between the modes consequently imposing a practical bandwidth limit on the minimum attainable coupling factor that varies inversely with the Quality factor of resonance at the fundamental modes of operation. Substituting this practical bandwidth limit enables the evaluation of the minimum measurable resolution in such sensors to be:

$$
\delta_{\min } \approx 8 \sum_{r=1}^{n} \sqrt{\frac{E_{t h} \Delta f}{2 E_{c} Q^{3} \omega_{r}^{e f f}}} .
$$

\section{ACKNOWLEDGMENTS}

The authors gratefully acknowledge the financial support from the British Council (UKIERI Grant No. SA06250), Churchill College and the Cambridge Commonwealth Trust. P. Thiruvenkatanathan thanks Mr. Luca Belsito for useful discussions.

${ }^{1}$ M. Li, H. X. Tang, and M. L. Roukes, Nat. Nanotechnol. 2, 114 (2007).

${ }^{2}$ X. L. Feng, C. J. White, A. Hajimiri, and M. L. Roukes, Nat. Nanotechnol. 3, 342 (2008).

${ }^{3}$ Y. T. Yang, C. Callegari, X. L. Feng, K. L. Ekinci, and M. L. Roukes, Nano Lett.6, 583 (2006).

${ }^{4}$ M. Su, S. Li, and V. P. Dravid, Appl. Phys. Lett. 82, 20 (2003).

${ }^{5}$ T. Thundat, E. A. Wachter, S. L. Sharp, and R. J. Warmack, Appl. Phys. Lett. 66, 1695 (1995).

${ }^{6}$ A. A. Seshia, M. Palaniapan, T. A. Roessig, R. T. Howe, R. W. Gooch,

T. R. Schimert, and S. Montague, J. Microelectromech. Syst. 11, 784 (2002).

${ }^{7}$ K. E. Wojciechowski, B. E. Boser, and A. P. Pisano, in 17th Ann. Int. Conf. Micro ElectroMech. Syst. (A MEMS resonant strain sensor operated in air, IEEE, Maastricht, The Netherlands, 2004) pp. 841-845.

${ }^{8}$ M. Spletzer, A. Raman, A. Q. Wu, X. Xu, and R. Reifenberger, Appl. Phys. Lett. 88, 254102 (2006).

${ }^{9}$ M. Spletzer, A. Raman, H. Sumali, and J. P. Sullivan, Appl. Phys. Lett. 92, 114102 (2008).

${ }^{10} \mathrm{P}$. Thiruvenkatanathan, J. Yan, J. Woodhouse, and A. A. Seshia, J. Microelectromech. Syst. 18, 1077 (2009).

${ }^{11} \mathrm{P}$. Thiruvenkatanathan, J. Yan, and A. A. Seshia, IEEE Trans. Ultrason. Ferroelectr. Freq. Control. 57, 690 (2010).

${ }^{12} \mathrm{P}$. Thiruvenkatanathan, J. Yan, J. Woodhouse, A. Aziz and A. A. Seshia, Appl. Phys. Lett. 96, 081913 (2010).

${ }^{13}$ E. Gil-Santos, D. Ramos, A. Jana, M. Calleja, A. Raman, and J. Tamayo, Nano Lett. 9, 4122 (2009).

${ }^{14}$ B. H. Tongue, Principles of Vibration (Oxford Univ. Press, New York, 2002), Vol. 2.

${ }^{15}$ T. B. Gabrielson, IEEE Trans. Electron Devices. 40, 903 (1993).

${ }^{16}$ N. M. M. Maia, J. M. M. Silva, J. He, N. A. J. Lieven, R. M. Lin, G. W. Skingle, W.-M. To, and A. P. V. Urgueira, Theoretical and Experimental Modal Analysis (Research Studies Press, Taunton, 1997), Vol. 1.

${ }^{17}$ S. H. Crandall and W. D. Mark, Random Vibration in Mechanical Systems (Academic Press, New York, 1963).

${ }^{18}$ M. V. Salapaka, H. S. Bergh, J. Lai, A. Majumdar, and E. McFarland, J. Appl. Phys. 81, 2480 (1997).

${ }^{19}$ K. L. Ekinci, Y. T. Yang, and M. L. Roukes, J. Appl. Phys. 95, 2682 (2004).

${ }^{20}$ K. Kokubun, M. Hirata, M. Ono, H. Murakami, and Y. Toda, J. Vac. Sci. Technol. A. 3, 2184 (1985).

${ }^{21}$ S. Pourkamali and F. Ayazi, Sens. Actuators A 122, 317 (2005).

${ }^{22}$ J. Yan, Ph.D. dissertation, University of Cambridge, 2007.

${ }^{23}$ J. Yan, A. A. Seshia, K. L. Phan, P. G. Steeneken, and J. T. M. van Beek, in Proc. 2007 IEEE Int. Freq. Control Symp. (Narrow bandwidth single-resonator MEMS tuning fork filter, IEEE, Geneva, Switzerland, 2007) pp. 1366-1369 . 